\title{
VULNERABILIDAD SÍSMICA DEL EDIFICIO CIENCIAS DE LA SALUD Y DEL SER HUMANO. UNIVERSIDAD ESTATAL DE BOLÍVAR - 2019
}

\author{
SEISMIC VULNERABILITY OF THE HUMAN AND HEALTH SCIENCES BUILDING. \\ STATE UNIVERSITY OF BOLÍVAR - 2019 \\ Elsa Moposita; Carlos Guaranga; María Mas; Gino Noboa \\ Universidad Estatal de Bolivar \\ Email:mmas@ueb.edu.ec \\ https://doi.org/10.33789/talentos.8.1.141
}

\begin{abstract}
Resumen: El territorio de Ecuador está expuesto a riesgo sísmico por su ubicación en la zona volcánica norte de la Cordillera de Los Andes, donde ocurren procesos tectónicos por los efectos de la subducción de la placa de Nazca bajo la placa Sudamericana. El riesgo sísmico se debe a tres factores: la amenaza sísmica, exposición y vulnerabilidad. La Universidad Estatal de Bolivvar está ubicada en la región sierra ecuatoriana, donde en los últimos años son más frecuentes los sucesos sísmicos, durante el evento de 2016 se visibilizaron daños parciales a la institución del campus matriz. La presente investigación surge con la finalidad de analizar la vulnerabilidad ante sismos del edificio en que radica la Facultad de Ciencias de la Salud $y$ del Ser Humano, con el propósito de determinar y ponderar las características actuales de la edificación e identificar el nivel de vulnerabilidad sísmica del mismo. Se realiza una investigación de campo en la que se aplica la ficha de la metodología aprobada por la Secretaria Nacional de Gestión de Riesgos de Ecuador para evaluación sísmica y verificación del nivel de vulnerabilidad física, se utiliza además el esclerómetro que aporta información respecto a la resistencia de la infraestructura. El nivel de vulnerabilidad de los factores físicos del edificio resulta de 36,6 puntos correspondiendo a un índice de vulnerabilidad sísmica medio; de los elementos estructurales estudiados se resume que el edificio tiene un desempeño aceptable pudiendo resistir ante la demanda de un evento sísmico.
\end{abstract}

Palabras Clave: Estructura, nivel de vulnerabilidad, riesgo sísmico, vulnerabilidad sísmica.

Recibido: 30 de marzo de 2020

Aceptado: 17 de marzo de 2021

Publicado como artículo científico en la Revista de Investigación Talentos VIII (1), 27-35 
Abstract: The territory of Ecuador is exposed to seismic risk due to its location in the northern volcanic zone of the Andes Mountains, where tectonic processes occur due to the effects of the subduction of the Nazca plate under the South American plate. Seismic risk is due to three factors: seismic hazard, exposure, and vulnerability. The State University of Bolivar is located in the Ecuadorian highlands region, where seismic events have been more frequent in recent years; during the 2016 event, partial damage to the institution of the parent campus was visible. The present investigation arises with the purpose of analyzing the vulnerability to earthquakes of the building in which the Faculty of Health Sciences and the Human Being is located, with the purpose of determining and weighing the current characteristics of the building and identifying the level of seismic vulnerability of the same. A field investigation is carried out in which the sheet of the methodology approved by the National Secretariat of Risk Management of Ecuador is applied for seismic evaluation and verification of the level of physical vulnerability, the sclerometer is also used that provides information regarding resistance of the infrastructure. The level of vulnerability of the physical factors of the building is 36.6 points corresponding to an average seismic vulnerability index; From the structural elements studied, it is summarized that the building has an acceptable performance, being able to withstand the demand of a seismic event.

Keywords: Structure, level of vulnerability, seismic risk, seismic vulnerability.

\section{INTRODUCCIÓN}

El territorio ecuatoriano está expuesto a riesgo sísmico por su ubicación en la zona volcánica norte de la Cordillera de Los Andes, donde ocurren procesos tectónicos por los efectos de la subducción de la placa de Nazca bajo la placa Sudamericana (Rivadeneira, 2007).

A partir del sismo del 16 de abril de 2016 ocurrido en el país, de amargas consecuencias sociales, económicas, estructurales, se incentivaron estudios en el tema de vulnerabilidad y riesgos sísmicos, los que eran escasos hasta entonces. Algunos referentes son los realizados en las ciudades como Cuenca y Quito. (Sigcho Gordillo \& Quizhpilema Piray, 2017) (Jiménez Pacheco,
Cabrera Cajamarca, Sánchez Beltrán, \& Avilés Tenorio, 2018).

La vulnerabilidad física está asociada a los daños que pueden sufrir los elementos estructurales de las edificaciones, lo que depende no solo de los materiales utilizados sino también de las características constructivas de la infraestructura. Se identifican estudios particulares al respecto en el país como es el desarrollado por Espinoza y colaboradores (Espinoza herrera \& Manzano, 2016) en el que se hace una evaluación similar.

Estudiar los riesgos a los que se encuentran expuestas las edificaciones facilita la actuación para minimizar estos a partir de 
las vulnerabilidades determinadas. Por otra parte identificar las estructuras acorde al cumplimiento o no de la aplicación de las Normas Ecuatorianas de Construcción (NEC) contribuye a toma de decisiones acertadas de las autoridades (Íñiguez, Barragán, Mas, \& Vallejo, 2017).

El riesgo sísmico se debe a tres factores: la amenaza sísmica, exposicióny vulnerabilidad: la amenaza sísmica, se relaciona con los movimientos telúricos que pueden ocurrir en un lugar; la exposición, se refiere a la cantidad de personas e infraestructuras que se encuentran en un lugar y pueden llegar a ser afectadas por la ocurrencia de un evento sísmico, y la vulnerabilidad que se relaciona con la capacidad de las edificaciones de resistir ante eventos sísmicos.

Los movimientos vibratorios que ocurren en el interior de la tierra durante un evento telúrico, suben a la superficie en forma de ondas sísmicas provocando daños en las infraestructuras, lo que ocasiona perjuicios o deterioros en las construcciones, que afectan su estética, su funcionalidad, o lo más grave, su seguridad estructural, siendo las infraestructuras solidas requerimientos clases para evitar desastres. (Amangandi Caspi, Yasuma Lazo, \& Aroca Barragán, Análisis de riesgos ante eventos sísmicos en las edificaciones de la parroquia SANTA FE, cantón GUARANDA, provincia BOLÍVAR, Ecuador., 2019) ((EL/ERS), 2016)

La vulnerabilidad sísmica de una estructura, grupo de estructuras o de una zona urbana se precisa como su predisposición intrínseca a sufrir daños ante la ocurrencia de un movimiento sísmico, está asociada directamente con las características físicas estructurales de las construcciones (Robet Diaz, 2016)

El daño en las edificaciones puede ser causado por fenómenos naturales, por la acción humana al darle un uso impropio a estas, así como también por una incorrecta construcción.

Para que las estructuras tengan un comportamiento satisfactorio frente a sismos fuertes es necesario que su diseño resista niveles de deformación más altos que los límites elásticos, debiendo ocurrir que ante sucesos de sismos moderados las estructuras no deben sufrir daños importantes que impidan su uso, y ante sismos severos no debe ocurrir el colapso de las edificaciones. Los análisis de vulnerabilidad requieren de la revisión del estado de la infraestructura lo que posibilita la realización posterior de un análisis de riesgos. (Secretaría CCT Foro Técnico COSIPLAN-IIRSA, 2016)

El estudio de la vulnerabilidad sísmica del hormigón armado y las mamposterías de las edificaciones se verifica mediante diversas técnicas e instrumentos, es el esclerómetro un instrumento que calcula un índice de rebote, que relaciona la dureza superficial del hormigón con su resistencia y la dureza.

Se conoce, según expertos, de las ventajas del uso del esclerómetro para evaluar vulnerabilidades estructurales por ser un ensayo no destructivo que permite 
realizar numerosas mediciones sin alterar la resistencia, estética y funcionalidad de las estructuras, además de ser un estudio económico y que permite ensayar numerosos elementos en un corto espacio de tiempo. Así como también tiene como requerimientos que sea aplicado en superficies lisas para realizar el ensayo (Web de informacion y formacion para profesionales y estudiantes, 2013).

Se dispone de estudios precedentes en edificaciones de la Universidad Central del Ecuador respecto al estudio de su estabilidad ante un evento sísmico con el fin de clasificar, seleccionar, describir las estructuras y los elementos correspondientes de acuerdo a los indicadores de riesgo sísmico, y saber si las edificaciones requieren estudios estructurales a detalle (Andrade, 2016).

La Universidad Estatal de Bolívar, en particular el edificio de la Facultad Ciencias de la Salud y del Ser Humano que se encuentra situado en el campus Alpachaca de la provincia de Bolívar Cantón Guaranda, fue construido en el año 2007. El inmueble cuenta con tres pisos levantados en un área de $381.23 \mathrm{~m}^{2}$; la edificación es de hormigón armado, mamposterías de bloques, paredes, columnas, vigas de hormigón armado, entre otros. Se sabe que los suelos sobre los que se yergue corresponden a limos arenosos, de plasticidad alta a media y consistencia baja (Poves Ferrer, 2015).

A la edificación afluyen cotidianamente estudiantes, docentes y demás personal administrativo de las carreras de Licenciatura en Enfermería y de Administración para
Desastres y Gestión del Riesgo, ascendiendo a un total de 1151 personas con flujo diario, lo que hace de interés identificar el estado de vulnerabilidad del inmueble.

Es objetivo de la investigación determinar el nivel de resistencia del hormigón y el grado de vulnerabilidad del edificio de la Facultad de Ciencias de la Salud y del Ser Humano de la Universidad Estatal de Bolívar.

\section{MATERIALES Y MÉTODOS}

En la investigación se utiliza la observación directa mediante una salida y recorrido de campo al lugar de estudio, se aplica la ficha de la matriz de la Metodología del Programa de Naciones Unidas para el Desarrollo, la cual está contenida en la metodología aprobada por la Secretaria Nacional de Gestión de Riesgos de Ecuador para evaluación sísmica y verificación del nivel de vulnerabilidad física en cada uno de los elementos estructurales del edificio (pilares, vigas, losas y gradas), la que toma como variables a las características físicas de la edificación (Secretaría Nacional de Gestión de Riesgos, 2016).

Para obtener el índice de vulnerabilidad física, se multiplica el valor del indicador por el peso de ponderación que da como resultado el máximo, la sumatoria de los valores máximos dan como producto final el índice, en cada uno de los indicadores, los que se califican en: 1 bajo, 5 medio, 10 alto; por último se hace la sumatoria de los valores y se determina el nivel de vulnerabilidad del edificio (Paucar Camacho, 2016).

Este valor calculado se clasifica según la 
propuesta Metodológica que para el análisis de vulnerabilidades está determinado por (SNGR-PNUD, 2012), la cual resume que de 0-33 de puntaje es Bajo, de 34 - 66 es Medio y más de 67 puntos es Alto, en referencia al nivel de amenaza sísmica.

Se realizan controles no destructivos en la edificación con la medición de la resistencia a la compresión de los elementos estructurales estudiados (Montenegro, 2011) .

Para la evaluación de pilares, vigas, losas y gradas de hormigón armado de realiza mediante métodos no destructivos al edificio mediante el uso del esclerómetro. Este instrumento permite medir la resistencia a la compresión del hormigón y realizar controles no destructivos de la calidad del mismo; el mismo ofrece un valor de rebote el cual se muestra en escala y establece las características del hormigón, con un enfoque hacia las patologías causadas por movimientos telúricos, resultado que se ofrece en porcentaje.

\section{RESULTADOS Y DISCUSIÓN}

En el estudio se determinó que la estructura se encuentra en un nivel de vulnerabilidad medio con 36.6 puntos (Tabla I), puntaje que sugiere la edificación tiene un desempeño estructural aceptable y puede resistir un evento de magnitud de menor grado, no obstante debe ser de seguimiento su comportamiento estructural. El resultado se clasifica acorde a lo estipulado por el SNGR-PNUD (2012) .

TABLA I.

EVALUACIÓN DEL ÍNDICE DE VULNERABILIDAD DEL EDIFICIO CIENCIAS DE LA SALUD, CON LA METODOLOGÍA DEL PNUD.

\begin{tabular}{|c|c|c|c|c|}
\hline \multicolumn{5}{|c|}{ EDIFICIO CIENCIAS DE LA S ALUD Y DEL SER HUMANO } \\
\hline \multicolumn{5}{|c|}{ COORDENADAS } \\
\hline \multicolumn{2}{|c|}{$\mathbf{X}$} & \multicolumn{3}{|c|}{721678.02} \\
\hline \multicolumn{2}{|c|}{$\mathbf{Y}$} & \multicolumn{3}{|c|}{982618.64} \\
\hline \multicolumn{5}{|c|}{ Índice de vulnerabilidad para amenazas sísmica } \\
\hline Indicadores & Previo amenazado & Amenaza sísmica & $\begin{array}{l}\text { Factor de pondera- } \\
\text { ción }\end{array}$ & $\begin{array}{l}\text { Puntaje ante sis- } \\
\text { mos }\end{array}$ \\
\hline Sistema estructural & Hormigón armado & 1 & 1.2 & 1.2 \\
\hline $\begin{array}{l}\text { Tipo de material de pare- } \\
\text { des }\end{array}$ & Pared de bloque & 1 & 1.2 & 1.2 \\
\hline Tipo de cubierta & Losa de hormigón armado & 1 & 1.2 & 1.2 \\
\hline Sistema de entrepisos & $\begin{array}{l}\text { Losa de hormigón } \\
\text { Armado }\end{array}$ & 5 & 1 & 5 \\
\hline Número de pisos & 3 pisos & 5 & 0.8 & 4 \\
\hline Año de construcción & entre 1991 y 2010 & 5 & 1 & 5 \\
\hline
\end{tabular}


Revista de Investigación Talentos, Volumen VIII (1), Enero - Junio 2021

ISSN Impreso: 1390-8197 ISSN Digital: 2631-2476

\begin{tabular}{|l|l|c|c|c|}
\hline Estado de conservación & Aceptable & 5 & 1 & 5 \\
\hline $\begin{array}{l}\text { Características del suelo de } \\
\text { la edificación }\end{array}$ & Firme- seco & 5 & 0.8 & 0,8 \\
\hline Topografía del sitio & A nivel de terreno plano & 5 & 0.8 & 4 \\
\hline Forma de construcción & Regular & 5 & 1.2 & 6 \\
\hline Nivel de vulnerabilidad física estructural del edificio ante amenaza símica & 36,6 \\
\hline
\end{tabular}

Fuente: Guaranda, C.; Moposita, E.

Para los indicadores de pilares, vigas, losas y gradas se promedian los valores obtenidos en la medición con el esclerómetro para cada elemento estructural del estudio, por plantas constructivas, medición que se resume porcentualmente para la edificación en general.

En la Tabla II se observa que coinciden, con un $33 \%$, la vulnerabilidad sísmica de planta baja y segunda planta alta con respecto a los pilares, siendo ligeramente mayor para la primera planta con solo un $1 \%$ más; el porcentaje indica una vulnerabilidad baja respecto a los pilares, lo cual sugiere un comportamiento estable de la edificación .

TABLA II

MATRIZ FINAL DE RESULTADOS DE ESTUDIO DE LA VULNERABILIDAD DE LOS PILARES. FACULTAD CIENCIAS DE LA SALUD Y DEL SER HUMANO.

\begin{tabular}{|l|c|c|}
\hline PILARES & $\begin{array}{l}\text { Presión de la } \\
\text { resisten cia } \\
\text { obtenida con } \\
\text { el escleróme- } \\
\text { tro } \\
\text { Kg/cm² }\end{array}$ & $\begin{array}{l}\text { Vulnerabilidad sísmica } \\
\text { del Edificio }\end{array}$ \\
\hline Planta Baja & 210 & $33 \%$ \\
\hline $\begin{array}{l}\text { P r i m e r a } \\
\text { Planta Alta }\end{array}$ & 215 & $34 \%$ \\
\hline $\begin{array}{l}\text { S e g u n d a } \\
\text { Planta Alta }\end{array}$ & 213 & $33 \%$ \\
\hline TOTAL & 638 & $100 \%$ \\
\hline
\end{tabular}

Fuente: Guaranda, C.; Moposita, E.
Para las vigas (Tabla III), se identifica la coincidencia de un $33 \%$ de vulnerabilidad sísmica, referente a este elemento de la planta baja y primera planta alta, siendo ligeramente mayor para la segunda planta alta con un $34 \%$; puntajes que sugieren la edificación tiene un comportamiento estable.

\section{TABLA III.}

MATRIZ FINAL DE RESULTADOS DE ESTUDIO DE LA VULNERABILIDAD DE LAS VIGAS.

FACULTAD CIENCIAS DE LA SALUD Y DEL SER HUMANO.

\begin{tabular}{|l|c|c|}
\hline VIGAS & $\begin{array}{l}\text { Presión de la } \\
\text { resistencia } \\
\text { obtenida con } \\
\text { el escleróme- } \\
\text { tro } \\
\text { Kg/cm² }\end{array}$ & $\begin{array}{l}\text { Vulnerabilidad sísmica } \\
\text { del Edificio }\end{array}$ \\
\hline Planta Baja & 212 & $33 \%$ \\
\hline $\begin{array}{l}\text { P r i m e r a } \\
\text { Planta Alta }\end{array}$ & 215 & $33 \%$ \\
\hline $\begin{array}{l}\text { S e g u n d a } \\
\text { Planta Alta }\end{array}$ & 218 & $34 \%$ \\
\hline TOTAL & 645 & $100 \%$ \\
\hline
\end{tabular}

Fuente: Guaranda, C.; Moposita, E.

El estudio del elemento estructural de losas se describe en la Tabla IV, observándose diferentes niveles de vulnerabilidad sísmica arrojando el menor valor en la planta baja (31\%). En la primera planta alta se identifica una vulnerabilidad media baja (solo 35\%), lo cual sugiere un comportamiento adecuado 
para la edificación.

TABLA IV

MATRIZ FINAL DE RESULTADOS DE ESTUDIO DE LA VULNERABILIDAD DE LAS LOSAS

FACULTAD CIENCIAS DE LA SALUD Y DEL SER HUMANO.

\begin{tabular}{|l|c|c|}
\hline $\begin{array}{l}\text { Losas del } \\
\text { Edificio }\end{array}$ & $\begin{array}{l}\text { Presión de la resis- } \\
\text { tencia de grada me- } \\
\text { dida con el escleró- } \\
\text { metro Kg/cm }\end{array}$ & $\begin{array}{l}\text { Vulnerabilidad } \\
\text { sísmica del Edi- } \\
\text { ficio }\end{array}$ \\
\hline Planta Baja & 215 & $31 \%$ \\
\hline $\begin{array}{l}\text { P r i m e r a } \\
\text { Planta Alta }\end{array}$ & 241 & $35 \%$ \\
\hline $\begin{array}{l}\text { S e g u n d a } \\
\text { Planta Alta }\end{array}$ & 228 & $33 \%$ \\
\hline TOTAL & 684 & $100 \%$ \\
\hline
\end{tabular}

Fuente: Guaranda, C.; Moposita, E.

La Tabla V muestra que la vulnerabilidad para las gradas del edificio tiene un mayor porcentaje $(51 \%)$ en la primera planta alta, siendo menor (49\%) en la planta baja. En ambos casos el valor es medio para la vulnerabilidad sísmica. Esto implica que, ante la presencia de un sismo, las gradas aún podrían resistir ante la demanda del evento. No obstante, las gradas son el elemento estructural de menor fortaleza del edificio bajo estudio, frente a la ocurrencia de un sismo.

\section{TABLA V}

MATRIZ FINAL DE RESULTADOS DE ESTUDIO DE LA VULNERABILIDAD DE LAS GRADAS

FACULTAD CIENCIAS DE LA SALUD Y DEL SER HUMANO.

\begin{tabular}{|l|l|l|}
\hline $\begin{array}{l}\text { Gradas del } \\
\text { Edificio }\end{array}$ & $\begin{array}{l}\text { Presión de la resis- } \\
\text { tencia de las gradas } \\
\text { medida con el escle- } \\
\text { rómetro Kg/cm }\end{array}$ & $\begin{array}{l}\text { Vulnerabilidad } \\
\text { sísmica del Edi- } \\
\text { ficio }\end{array}$ \\
\hline Planta Baja & 241 & $51 \%$ \\
\hline $\begin{array}{l}\text { P r i m e r a } \\
\text { Planta Alta }\end{array}$ & 234 & $49 \%$ \\
\hline
\end{tabular}

\begin{tabular}{|l|l|l|}
\hline TOTAL & 475 & $100 \%$ \\
\hline
\end{tabular}

Fuente: Guaranda, C.; Moposita, E.

Los resultados que se muestran en el presente trabajo son obtenidos de forma similar a los utilizados en el estudio de (Amangandi Caspi, Yasuma Lazo, \& Aroca Barragán, 2019) en el análisis de riesgos que ante eventos sísmicos se realizó para las viviendas de la comunidad Shunguna, Santa Fé, cantón Guaranda, Provincia de Bolívar,

\section{CONCLUSIONES}

El nivel de vulnerabilidad de los factores físicos del edificio resulta de 36,6 puntos correspondiendo a un índice de vulnerabilidad sísmica medio acorde a la metodología de evaluación de riesgo sísmico en edificaciones del PNUD. La edificación tiene un desempeño aceptable y puede resistir un evento de magnitud de menor intensidad, no obstante se sugiere el seguimiento del comportamiento estructural de la edificación.

Se definen como los elementos estructurales para el estudio de vulnerabilidad a pilares, vigas, gradas y losas, de los que se identificaron dos elementos con vulnerabilidad media en la primera planta alta correspondiendo a pilares (34\%) y losas (35\%); las vigas presentan también vulnerabilidad en la segunda planta alta (34\%) así como en las gradas de la planta baja $(51 \%)$.

Las gradas son el elemento estructural de menor fortaleza del edificio bajo estudio, frente a la ocurrencia de un sismo.

Como conclusión más importante se dice que el edificio tiene un desempeño aceptable pudiendo resistir ante la demanda de un 
evento sísmico.

\section{REFERENCIAS BIBLIOGRÁFICAS}

(EL/ERS), A. R. (25 de 08 de 2016). $\mathrm{La}$ infraestructura es clave para evitar desastres. Obtenido de https://www.dw.com/es/lainfraestructura-es-clave-paraevitar-desastres/a-19503093

Amangandi Caspi, K., Yasuma Lazo, E., \& Aroca Barragán, G. (2019). Análisis de riesgos ante eventos sísmicos en las edificaciones de la parroquia SANTA FE, cantón GUARANDA, provincia BOLÍVAR, Ecuador. Revista de Ciencias de Seguridad y Defensa, 50 - 88 .

Andrade, D. \&. (2016). Evaluación de la vulnerabilidad sísmica del Edificio de la Facultad de Comunicación Social, de la Universidad Central del Ecuador, utilizando la Norma Ecuatoriana de la Construcción (NEC-SE-RE, 2015). . Quito: UCE.

Espinoza herrera, J., \& Manzano, J. (2016). Estudio de la vulnerabilidad sísmica de una unidad educativa. Caso de la Escuela Primaria de la Armada nacional. Guayaquil Ecuador.: RepositorioUniversidaddeGuayaquil. Íñiguez, G., Barragán, G., Mas, M., \& Vallejo, M. (2017). Estudio de amenazas, vulnerabilidad y riesgos estructurales en las instituciones de la parroquia urbana del Cantón Chimbo. Publicando, 554-562.
Jiménez Pacheco, J., Cabrera Cajamarca, J., Sánchez Beltrán, J., \& Avilés Tenorio, F. (2018). Vulnerabilidad sísmica del patrimonio edificado del Centro Histórico de la Ciudad de Cuenca: Lineamientos generales y avances del proyecto. MASKANA, 59-78.

Montenegro, J. (27 de septiembre de 2011). Evaluación de estructuras con el uso de esclerómetro. Recuperado el 30 de diciembre de 2019, de https://civilgeeks.com/2011/09/27/ evaluacion-de-estructuras con-el-uso-de-esclerometro/

Paucar Camacho, J. A. (2016). Metodologia vulnerabilidad fisica de las edificaciones. Guaranda: UEB.

Poves Ferrer, F. (2015). Exposición de las patologías más habituales en los Edificios. Cantabria: Colegio de arquitectura tecnica de Cantabria.

Rivadeneira, F. (2007). Breves fundamentos sobre lso terremotos en el Ecuador. Quito: Instituto Geofisico de la Escuela Politecnica Nacional .

Robet Diaz, R. (2016). Vulnerabilidad y daños sismicos: concepto y evaluación. Obtenido de Repositorio Universidad de LLeira: https://www.tdx.cat/bitstream/ handle/10803/6230/03CAPITULO2. pdf? sequence $=3 \&$ is Allowed $=y$

Secretaría CCT Foro Técnico COSIPLANIIRSA. (11 de 2016). La Gestión de Riesgos de Desastres en COSIPLAN: Medidas de reducción de riesgo 
en infraestructura de integración

de Chile y Perú. Buenos Aires, Argentina: UNASUR- COSIPLAN.

Obtenido de http://www.iirsa. org/admin_iirsa_web/Uploads/ Documents/2_Informe_GDRERN_Interior_BAJA_completo.pdf

Secretaría Nacional de Gestión de Riesgos. (2016). Guía práctica para evaluación sísmica y rehabilitación de estructuras, de conformidad con la Norma Ecuatoriana de la Construcción NEC 2015. Quito: Secretaria Nacional de Gestión de Riesgos (SNGR).

Sigcho Gordillo, M., \& Quizhpilema Piray, A. (2017). Evaluación de la vulnerabilidad sísmica del edificio de aulas de la Facultad de Ingeniería de la Universidad Central del Ecuador, utilizando la Norma Ecuatoriana de la Construcción (NEC-SE-RE, 2015). Quito, Ecuador : Repositorio de la Universidad Central del Ecuador.

SNGR-PNUD. (2012). SNGR PNUD, S. (2012). Propuesta Metodologica Analisis de Vulnerabilidades a Nivel Municipal. ISBN. 99429887-4. Quito, Ecuador: SNGR.

Web de informacion y formacion para profesionales y estudiantes. (2013). Patologia Rehabilitacion y Construccion . Recuperado el 30 de diciembre de 2019, de https://www. patologiasconstruccion.net/2013/11/ resistencia-del-hormigon-mediantees clerometro-o-indice-de-r 